\title{
Kernos
}

Revue internationale et pluridisciplinaire de religion grecque antique

16 | 2003

Varia

\section{Les religions du monde ancien : entre typologie et} histoire

À propos d'un article récent

Maria Grazia Lancellotti

\section{(2) OpenEdition \\ Journals}

Édition électronique

URL : http://journals.openedition.org/kernos/829

DOI : $10.4000 /$ kernos.829

ISSN : 2034-7871

Éditeur

Centre international d'étude de la religion grecque antique

\section{Édition imprimée}

Date de publication : 1 janvier 2003

Pagination : 225-246

ISSN : 0776-3824

\section{Référence électronique}

Maria Grazia Lancellotti, « Les religions du monde ancien : entre typologie et histoire », Kernos [En ligne], 16 | 2003, mis en ligne le 14 avril 2011, consulté le 01 mai 2019. URL : http:// journals.openedition.org/kernos/829 ; DOI : 10.4000/kernos.829 


\title{
Les religions du monde ancien : entre typologie et histoire
}

\author{
À propos d'un article récent ${ }^{*}$
}

\author{
Non domandarci la formula che mondi possa apritti \\ si qualcbe storta sillaba e secca come un ramo \\ Codesto solo oggi possiamo dirti, \\ ciò che non siamo, ciò che non vogliamo. \\ Non cbiederci la parola, Eugenio Montale
}

La parution d'un article expressément consacré à la méthodologie de l'histoire des religions est un événement d'une certaine rareté, dans le panorama scientifique italien des dernières années, où l'on manifeste en général peu d'intérêt ${ }^{1}$ pour le débat qui caractérise notre discipline à l'époque post-moderne ${ }^{2}$, tout à fait critique en ce qui concerne la recherche d'universels ${ }^{3}$.

Dans ce cadre vient s'insérer une tentative récente de F. Mora qui propose une typologie des religions "classiques " ${ }^{4}$, en abordant en plus une série de problèmes anciens, délicats et complexes, qui vont de la délimitation de l'objet d'étude jusqu'à l'élaboration de nouveaux outils heuristiques pour affiner les méthodes de recherche. Une fois reconnus à l'auteur du courage et de la bonne volonté, il convient néanmoins de ne pas laisser passer l'occasion d'intervenir dans ce débat - ouvert implicitement - et de se mesurer avec ces propositions dont il faudra examiner soigneusement les prémisses, la nouveauté et les progrès éventuels pour l'histoire des religions.

Dans ce but, il est opportun de résumer la pensée de l'auteur le plus fidèlement possible et de tirer au clair les points forts de son argumentation. En même temps, je proposerai quelques remarques qui concernent certains aspects primordiaux de son

* F. Mora, "Verso una tipologia delle religioni classiche", Kernos 13 (2000), p. 9-33 (= Mora 2000). Je remercie le prof. Paul-Eugène Dion qui a eu l'amabilité de revoir le français de cet article.

1 Pour se convaincre, il suffit de consulter la bibliographie du bulletin Science of Religion Abstracts and Index of Recent Articles, les derniers numéros des revues Revue de l'bistoire des religions, History of Religions, Temenos, Numen, Metbods E Theory in the Study of Religion et aussi Studi e materiali di storia delle religioni, ainsi que les actes des divers colloques régionaux ou mondiaux promus par l'International Association for the History of Religions.

2 En général, $c f$. (entre autres) Bauman 1993; Segal - Ryba 1997; Cox 1998. Voir aussi les articles sur la méthode dans Numen 48 (2001).

3 En ce qui concerne les dernières années et les volumes (individuels ou collectifs) les plus récents, voir entre autres Saler 1993 (et le «Symposium » sur son livre dans Geertz - McCutcheon 2000a, p. 287-338 [= MTSR 12, 2000]); Klimkeit 1997; Boespflug - Dunand 1997; Idinopoulos - Wilson 1998; Taylor 1998; Platvoet - Molendijk 1999; Braun - Henking 1999; Wiebe 1999; Geertz McCutcheon 2000a; Feil 2000. Voir aussi le compte rendu général par McCutcheon 1995, ainsi que le bilan du débat international au sein de la IAHR dressé par Geertz - McCutcheon $2000 \mathrm{~b}$.

4 Sur l'acception particulière à la base de cette définition, $c f$. infra. 
étude en m'efforçant d'indiquer des voies de recherches différentes et/ou alternatives, guidées par des principes méthodologiques qui ne coïncident pas avec ceux qui sont à la base de l'approche de Mora. Tout ceci, bien entendu, dans un esprit constructif aussi bien dans la prise de distance que dans l'espoir que la discussion puisse s'élargir et stimuler d'autres contributions.

Je me propose en particulier d'aborder les thèmes suivants :

a) la validité des critères proposés par Mora et qui permettraient de déterminer le terrain spécifique où l'historien des religions doit travailler;

b) ses propositions concernant la méthodologie à adopter;

c) l'utilité d'une classification «typologique » générale et, en particulier, d'une typologie appliquée aux religions dites « classiques » telle que celle proposée par Mora;

d) la possibilité d'inclure dans cette dernière définition toutes les religions de la Méditerranée et de l'Asie occidentale, antérieures au monde grec et romain.

Mora développe son argumentation en trois parties principales. Dans la première partie, il se propose de fournir une définition de l'objet et de la méthode de l'histoire des religions; dans la seconde partie, il indique des paramètres pour élaborer une «typologie » générale des religions fondée sur les sources disponibles; dans la troisième partie, enfin, il applique cette «typologie » aux religions «classiques», une définition qui inclut toutes les religions européennes et proche-orientales du monde ancien, tant pré-classiques que classiques.

Dans un premier paragraphe intitulé «La Storia delle religioni: oggetto e metodo», Mora examine la question primordiale de la « definibilità dell'oggetto della materia, quale necessaria premessa scientifica dell'indagine storico-religiosa » (p. 9). À cet égard, le problème fondamental serait celui de l'universalité et de la spécificité de l'expérience religieuse. Pour citer les mots de l'auteur, "Si tratta cioè di stabilire da un lato se sia possibile definire un modello unico, generalissimo, di religione per procedere allo studio universale della Religione [c'est moi qui souligne], dall'altro se si possa individuare una struttura fondamentale del comportamento religioso, come ambito più specifico del comportamento umano, individuale e collettivo » (p. 9)

En abordant la première question, celle de la possibilité de définir l'objet de la discipline, Mora indique comme point de repère capital la position d'Ugo Bianchi sur l'interprétation spécifique des faits religieux, qui permettrait de dépasser l'opposition entre les défenseurs de l'existence $a b$ aeterno d'une structure religieuse de $l^{\prime h o m m e}{ }^{6}$ et ceux qui estiment comme inévitable la "dissoluzione dell'oggetto

5 Il n'est pas possible de discuter ici dans tous les détails la forme et la substance de cette affirmation, où Mora met en cause d'une manière discutable deux problèmes capitaux, celui de la possibilité de définir l'objet de la discipline et celui de sa méthode particulière. Tandis que ces deux questions constituent les thèmes fondamentaux de l'histoire des religions et il ne s'agit aucunement d'une découverte de Mora, il faut remarquer que « lo studio universale della Religione » ne peut absolument pas représenter l'objectif d'une discipline à orientation historique.

6 Identifiés par Mora avec les phénoménologues « classiques » (R. Otto, G. van der Leeuw, M. Eliade et aussi J. Ries), à côté desquels il mentionne aussi - d'une manière étonnante E. Durkheim, dont l'approche ne peut pas être mise sur le même plan que celle des autres savants qui, même si chacun sto modo, rentrent tous dans l'école phénoménologique. 
religioso » (mais Mora le définit en tant qu'« oggetto storicoreligioso », ce qui n'est pas exactement la même chose), représentés en Italie surtout par D. Sabbatucci ${ }^{7}$

À ce sujet, il faut remarquer que l'histoire des études - aussi bien anciennes que contemporaines - n'autorise guère un cadre si radicalisé et simpliste des différentes tendances méthodologiques. Entre les deux «partis» indiqués par Mora comme " extrêmes », beaucoup de savants se situent sur des positions plus équilibrées: d'une part, on n'admet pas que l'on puisse parler de « Religion » avec la majuscule et au singulier; d'autre part, on estime qu'il n'existe aucune structure religieuse présente $a b$ aeterno dans l'homme ni non plus que l'histoire des religions risque de perdre son objet par suite de cette prise de position.

La position de Sabbatucci lui-même, considérée comme extrême dans ce sens, doit être en effet relativisée et mise sur le même plan que les approches de plusieurs ethnologues et sociologues de la religion : tous sont d'accord - même si chacun d'un point de vue différent - pour considérer la religion comme une manifestation ou une partie de la culture, qu'il est difficile de distinguer comme objet spécifique de recherche. À côté de ces derniers l'on trouve d'autres historiens des religions, comme p. ex. K. Rudolph ${ }^{8}$, qui estiment que la recherche historico-religieuse possède bien sûr un objet et une méthode spécifiques, mais qu'elle doit se développer à l'intérieur des sciences historiques sans s'aventurer à la recherche de modèles abstraits, de structures idéales, d'essences ontologiques.

Comme on vient de le remarquer, Mora estime qu'entre les deux approches « extrêmes » il en est une troisième, élaborée par U. Bianchi, susceptible d'offrir une perspective théorique différente et privilégiée. Selon cette approche, il y aurait des « significative continuità parziali tra singole religioni, che permettono (nel caso limite) di riscontrare una sostanziale affinità anche tra religioni non direttamente confrontabili, ma che risultino a loro volta comparabili con un terzo elemento » (p. 10). Entre la thèse d'une structure religieuse unique connaturelle à l'homme et présente dans toute forme d'humanité, et celle qui finit par dissoudre l'objet religieux dans le domaine plus général de la culture, la position de Bianchi, fondée sur le concept d'analogie ${ }^{9}$, est considérée par Mora comme la seule voie possible à parcourir pour sortir de toute contradiction et concilier le général avec le particulier.

La position de Bianchi, dont l'exposition détaillée demanderait un travail spécifique, est évoquée par Mora d'une façon rapide et quelque peu apodictique, et entraîne en plus un saut logique qui finit par escamoter le problème de la définition de la religion. De toute façon, il s'agit d'un problème qui revient inévitablement à cause de l'emploi non circonstancié de l'adjectif « religieux», utilisé pour caractériser des faits ou des phénomènes divers mais apparentés par un présumé « air de famille » («somiglianza familiare ») qui fait son entrée ex abrupto. Tandis que dans l'approche de Bianchi cette voie de recherche, acceptable ou non ${ }^{10}$, est de toute façon élaborée à l'intérieur d'une réflexion systématique, le rappel de Mora a plutôt l'aspect d'un acte

7 La seule mention de l'article Kultur und Religion rédigé par D. Sabbatucci dans le cadre du Handbucb religionswissenschftlicber Grundbegriffe (éd. par H. Cancik, B. Gladigow, M. Laubscher) I, Stuttgart, 1988, p. 43-58, ne rend pas justice aux analyses plus approfondies de ce savant dont il faut au moins rappeler Sabbatucci 1987 et 1990.

8 Tout à fait exemplaires dans ce sens sont les remarques de Rudolph 1985b, formulées et approfondies clans de nombreux travaux, parmi lesquels $c f$. en particulier Rudolph 1992.

9 Cf. entre autres Bianchi 1970, p. 3-27; Bianchi 1975; Bianchi 1983, p. 17-28.

10 Pour une évaluation critique, $c f$. p. ex. Geertz 1997, en particulier p. $35 s q$. 
de foi insuffisamment médité : il présente cette démarche comme le choix définitif, sans aucune préoccupation de le motiver et de l'expliquer convenablement.

Après ces prémisses, Mora remarque que l'objet de la discipline serait « in via primaria l'insieme dei fenomeni soggettivamente esperiti come religiosi [c'est moi qui souligne] ed in via secondaria quei fenomeni che, per la loro analogia con quelli percepiti come religiosi in altre civiltà, possono essere ugualmente considerati religiosi, pur non essendo soggettivamente esperiti come tali [c'est moi qui souligne], ed in particolare tra essi i residui e le forme attenuate di religiosità » (p. 1011). Ces affimations soulèvent diverses objections dont deux au moins, de nature substantielle, doivent être formulées explicitement. La première objection concerne la cohérence logique et formelle de ces thèses; la deuxième concerne une option de méthode qui rend sérieusement perplexe à différents niveaux.

Tout d'abord, une perplexité substantielle concerne les deux phases du processus qui permettrait de repérer les «faits religieux». Dans une première phase, le chercheur est censé reconnaitre le caractère « religieux » des phénomènes sur la base de sa propre expérience (« esperire soggettivamente ", selon Mora); dans une deuxième phase, il doit réussir à saisir une autre série de faits par une opération qui, à défaut de se situer au dehors de son expérience, devrait le mettre en condition de découvrir les analogies entre ces derniers faits et les autres, reconnus dans la phase précédente.

S'il est difficile de passer sous silence le manque de rigueur d'une théorie qui prétend se fonder sur le penchant subjectif du chercheur et se caractérise par le manque d'intérêt pour la question des conditionnements culturels (les risques de l'ethnocentrisme) ${ }^{11}$, on n'arrive pas à comprendre les stratégies mises en cuvre par le chercheur afin de reconnaître, dans la deuxième phase, des faits religieux qui, au point de départ, n'étaient pas à la portée de l'expérience du sujet. Ne s'agit-il pas toujours du même sujet qui met en œuvre ses ressources techniques et intuitives dans les deux phases? Comment peut-on saisir autrement les analogies parmi des faits / phénomènes divers, sans avoir toujours recours à ses propres critères d'interprétation? De toute évidence, les deux « phases » ne peuvent pas être distinguées l'une de l'autre ni sur le plan de la logique, ni sur celui de la méthode, puisqu'il s'agit toujours du même sujet qui réfléchit et interprète. Toutes ces remarques ne se posent bien sûr qu'au niveau de la cohérence interne de la théorie de Mora, dont on attendrait plutôt un énoncé rigoureux du point de vue épistémologique et formel.

La deuxième objection concerne l'élimination radicale de ce processus cognitif du point de vue « emic », interne à chaque culture. Selon Mora, les paramètres culturels des différentes sociétés sous enquête ne jouent aucun rôle et n'ont qu'une importance marginale dans le type de recherche qu'il théorise. Or, chacun sait que le problème des relations entre la perspective (inévitablement ethnocentrique) du chercheur et celles des membres appartenant aux cultures «autres » est aujourd'hui au centre d'un vaste débat dont Mora ne semble avoir aucune perception. Il s'agit de la dialectique (bien connue) entre les points de vue « emic » et « etic ${ }^{12}$, tous les deux impliqués à des niveaux différents dans les processus d'interprétation. Sans arriver à

11 L'attention prêtée par Mora à ce problème fondamental dans sa discussion sur la typologie des sources me paraît insuffisante (cf. infia): il faudrait l'aborder comme une question préliminaire et primordiale de la méthode historico-religieuse, comme il ressort de la vaste bibliographie récente à ce propos (résumée par Saler 1993, p. 227-264).

12 Cf. entre autres les différentes contributions dans Headland - pike - Harris 1990 et, dernièrement, Geertz 2000, en particulier p. $70 s q$. 
utiliser (même à titre d'expérience) des catégories conceptuelles empruntées aux cultures « autres » - comme on l'a suggéré ${ }^{13}$ - il est étonnant, du point de vue méthodologique, d'ignorer sic et simpliciter la question insider/outsider, sur laquelle une littérature imposante s'est développée ${ }^{14}$.

Quelle que soit la position que l'on assume face à ces problèmes clélicats, il est tout à fait clair que la solution la moins appropriée consiste à les sous-estimer ou à les considérer comme résolus. Au lieu d'« esperire soggettivamente »-il ne me semble pas s'agir ici d'une question purement terminologique - l'historien des religions, sur la base de sa formation spécifique, doit plutôt faire appel à une compétence qui puisse faire de lui un observateur privilégié des faits à analyser. De toute façon, il ne peut absolument pas échapper à une confrontation, rigoureuse et systématique, avec les cultures qu'il étudie de son point de vue particulier : cultures qu'il devra connaitre (philologiquement, historiquement) le mieux possible, en particulier en ce qui concerne leurs valeurs, leurs expériences, en un mot, leur système idéologique et culturel.

En laissant de côté pour l'instant d'autres implications issues de ces prémisses, examinons l'articulation du modus operandi de l'histoire des religions, telle qu'elle est conçue dans cet article. Le processus opérationnel de cette discipline consisterait donc en la «produzione di una complessa tipologia storica delle religioni e dei motivi religiosi » (p. 11), à orienter, en ce qui concerne les « religioni », vers « il principium individuationis di una singola civiltà o ambiente più specifico » et, en ce quí concerne les « motivi religiosi », vers « il grado di generalità (piuttosto che di universalità) di determinati 'ingredienti' del fenomeno religioso » (p. 11).

En dépit de l'emploi de l'adjectif « historique » appliqué à la typologie ( $c$. infra), il y a ici le risque concret que la phénoménologie, expulsée par la porte, puisse rentrer par la fenêtre. L'élaboration des "typologies ", même bâties sur les données historiques, implique inévitablement l'emploi d'une classification abstraite qui n'a de validité qu'au niveau heuristique, puisque elle vise à une description et un classement systématique de «types" ou "modèles» (plusieurs définitions sont admissibles) souvent utiles, mais qui ne représentent certes pas l'objectif ultime de la recherche.

En suivant l'argumentation de Mora, qui ne nous explique guère la différence entre «tipi », « motivi religiosi » et « ingredienti »du «phénomène religieux », l'on arriverait de la sorte à une « définition » de la religion qui vaudrait aussi comme outil d'orientation : elle consiste précisément dans l'« idea che la religione rappresenti, produca, costituisca una rottura di livello, cioè l'introduzione di un elemento 'altro' dalla realtà esperita quale elemento essenziale dell'esperienza » (p. 11). Il faut bien réflechir sur le sens et la portée de ces affirmations, puisqu'on arrive par là au noyau substantiel de la pensée de Mora. De façon intuitive l'on arriverait à percevoir que la religion se caractérise et se manifeste par une « rottura di livello » (litt. : rupture de niveau) qui intervient dans l'expérience humaine, où un autre élément s'introduit en produisant une transformation radicale du type de réalité expérimentée jusqu'à ce moment-là. Comme Mora l'affirme explicitement, l'on constate de la sorte que « la religione si caratterizza come rottura di livello rispetto alla totalità di una cultura [c'est moi qui souligne] » (p. 11).

Ici Mora se réfère à une « altérité » par rapport à la réalité expérimentée, une altérité qui entre dans l'expérience et la marque profondément ou bien, comme il

13 C'est, par ex., le reproche adressé à B. Saler par E.W. Paden dans Geertz - McCutcheon $2000 a$, p. 307-313.

lá Cf. supra, n. 12 et encore, p. ex., McCutcheon 1999. 
l'explique dans une note, qui est, dans sa forme la plus atténuée, « una diversa percezione della realtà esperibile ». Mais quel sens faut-il attribuer à cette " altérité ", à cette « diversità di percezione »? Comment peut-on la repérer dans des contextes culturels où les termes et les catégories conceptuelles de «religion » et de «sacré » n'existent pas ou bien ne correspondent pas aux nôtres? Ne s'agit-il pas plutôt de la projection sur des civilisations différentes d'un concept de «transcendant » qui n'appartient qu'à notre culture? Le problème est loin d'être neuf, mais il est toujours fort contraignant. En réalité, il y a une différence fondamentale entre le choix de situer cette "rupture de niveau " dans le domaine de la réalité expérimentée (question qui, à la rigueur, concerne beaucoup plus le philosophe que l'historien), que de la placer par contre sur un plan extra- ou méta-culturel ${ }^{15}$. Cette deuxième option empêche évidemment toute analyse historique qui ne peut être menée que dans le domaine de la culture humaine. Dans les deux cas, de toute façon, l'enquête historique est malmenée ou carrément abolie.

À ce stade, il est légitime de s'interroger sur les critères permettant d'établir quand, comment, où et par rapport à quelle notion de culture cette « rupture de niveau » peut être vérifiée. Cela se passe-t-il d'une façon intuitive, où bien existe-t-il des symptômes « objectifs »? Dans l'affirmative, comment peut-on les identifier? Sont-ils valides pour les religions historiques, ou bien faut-il les rechercher comme des « universels » présents dans toutes les cultures ? À cet égard, on reste dans le noir le plus complet. La tâche du spécialiste est rendue encore plus ardue par le fait que, comme on vient de le remarquer, les "voix » de chaque culture, le point de vue « emic », ne reçoivent pas la moindre attention. Pour Mora, semble-t-il, il n'est pas question d'analyser cette sorte de metabasis eis allo genos (la prétendue « rupture de niveau ») en travaillant aussi sur les valeurs caractéristiques de chaque société : le savant est censé ne les saisir qu'à travers un processus intérieur qui se pose évidemment sur un plan subjectif et intuitif, une « méthode », soit dit en passant, difficilement compatible avec une approche qui se veut scientifique. Comme Th. P. van Baaren (entre autres) l'a remarqué, "Intuition is certainly important, but appeal to it is not an argument in any scientific discussion $\gg 16$.

Il faut encore ajouter que la note 13 de l'article de Mora, apparemment une glose secondaire de son argumentation, montre une incohérence interne tout à fait frappante : ici on parle en effet d'une « diversa percezione della realtà esperibile » comme témoignage de la « rottura di livello », mais cette affirmation finit au bout du compte par reconduire (sans doute, inconsciemment) la religion dans le domaine des expériences humaines, marqué par une différence qui n’est pas de qualité par tapport aux autres domaines.

Or, il est nécessaire de prendre une décision de fond. Ou bien on déclare ouvertement que la religion - avec la prétendue « rupture de niveau » qu'elle « rappresenta, produce e costituisce »- est un phénomène méta-culturel, et dans ce cas l'historien est clésarmé; ou bien on admet que la religion fait partie de la culture humaine comme manifestation particulière de cette dernière, mais au même titre que les autres manifestations. Dans ce deuxième cas, le seul concevable pour une histoire des religions conçue comme discipline scientifique, la « rupture de niveau » devra être abandonnée dans toutes ses implications méta-historiques et, tout au plus, pourra-t-

15 Il ne me semble pas pertinent de citer à ce propos Platvoet 1994, une contribution dont il existe de toute façon une version plus longue et détaillée (Platroet 1990).

16 Van Baaren 1973, p. 49. 
elle être réintégrée comme une parmi les nombreuses définitions opérationnelles dont le but n'est que de permettre une étude plus approfondie du phénomène « religion ». À ce moment, le rôle de l'observateur est ramené drastiquement (et sagement!) au plan concret de l'enquête historico-religieuse, rivée à l'étude de la religion en tant que manifestation culturelle abordable par une méthodologie spécifique.

Mais approfondissons davantage les implications de ce concept de « rupture de niveau » et de sa définition. Bien que Mora parle du « carattere funzionalistico » de cette définition car elle « non individua (...) una forma generalissima, ma soltanto un'analogia di funzione rispetto all'insieme dei dati antropologici, al cui interno possono darsi elementi di discontinuità più o meno forti, ma mai di totale divergenza » (p. 11), il n'est pas difficile de découvrir que le choix de postuler l'existence, toujours et partout, d'une « rupture de niveau » comme aspect caractéristique d'une série de phénomènes, implique ipso facto une évaluation sui generis de la religion précisément comme un ganz Anderes, abstraction faite de ses différentes manifestations. Ici, il faut le remarquer, le savant est disposé à admettre que «il grado in cui si compie la rottura di livello potrà essere diverso, così come la materia impegnata e la forma (maggiore o minore distinzione di sfere separate) », étant donné que « la polarità tra dati antropologici empiricamente riscontrabili, l'elemento o gli elementi cui è affidata la rottura di livello e l'insieme rappresentato da ogni singola cultura rende d'altronde ragione della difficoltà di ridurre ad un minimo comun denominatore il concetto di religione $»$ (p. 11).

Que l'on ait affaire au véritable noud du problème est indiqué par l'affirmation que « $(\ldots)$ la religione si caratterizza come rottura di livello rispetto alla totalità di una cultura (o sub-cultura) » (ibid.). Tandis que Mora avait parlé peu avant de rupture de niveau par rapport à la réalité expérimentée, la référence est ici carrément à la culture. Encore une fois, donc, on réaffirme le concept de la religion comme quelque chose d'autre par rapport à la culture humaine, quelque chose qui la casse, la dépasse, la transcende, ce qui a d'énormes conséquences et implications pour notre discipline et sa vocation historique.

Il y a, à ce stade-ci, un passage qui concerne la notion d'expérience religieuse, conçue " come un contimuum polarizzato (...) intorno ad alcune opzioni fondamentali » (p. 12), concept qui pousse l'auteur à avoir recour's à une approche nouvelle, à la frontière entre histoire des religions et disciplines apparentées. Selon Mora, le caractère interdisciplinaire typique de l'histoire des religion serait dû au « carattere prevalentemente funzionale della 'rottura di livello » : d'où les phénomènes de " double appartenance », phénomènes qui - si je comprend bien - produisent une rupture de niveau mais rentrent dans le domaine d'autres disciplines à cause de la forme spécifique d'activité humaine qu'ils présupposent.

Il ne me semble pas qu'on ait affaire à des problèmes capitaux de (et même pas pertinents à) la méthode historico-religieuse, comme il ressort par exemple de la difficulté de définir et de déterminer la « rupture de niveau ». Il est évident que le concept de « rupture de niveau » ne fait que reproduire - dans des termes différents la question de la définition de l'objet de la discipline, puisqu'il est considéré comme le critère décisif pour distinguer ce qui est « religieux » de ce qui ne l'est pas. Il n'y a rien d'étonnant, de la sorte, dans le fait que l'application de ce critère puisse provoquer tant de difficulté, étant donné la prétention de sortir du domaine de la culture pour aller à la recherche d'un « religieux » qui, loin d'être considéré comme une manifestation culturelle, est censé faire partie d'une tout autre dimension. Si, par contre, on accepte de faire rentrer la religion dans la sphère des faits culturels 
(comme cela me paraît inévitable) sans arriver en même temps à l'éliminer comme objet de recherche, la perspective change radicalement : on n'a plus affaire au problème d'une "superposition » ("sovrapposizione ») mais, tout simplement, à celui des différentes optiques - de discipline et de méthode - d'après lesquelles on aborde l'étude de l'histoire humaine.

Il est sans doute superflu de rappeler que chaque discipline - ici on se réfère précisément aux sciences dites humaines - a bien sûr le droit de revendiquer son autonomie, mais elle peut être à la fois auxiliaire d'autres disciplines, selon les optiques adoptées et les méthodes choisies par le chercheur, d'après sa formation et les objectifs qu'il se pose dans ses enquêtes. On ne voit pas pourquoi l'histoire des religions devrait échapper à cette interaction dialectique avec les autres disciplines, ou bien représenter une exception. Si l'histoire des religions peut apparaître " marcatamente interdisciplinare » (mais a-t-elle vraiment ce caractère plus que la recherche historique fondamentale?), cela n'est pas dû au « carattere funzionale della rottura di livello », mais au fait qu'il s'agit d'une discipline historique qui, comme telle, a comme toile de fond unique et complexe la culture dont elle fait partie et dont elle représente - tout à fait comme les autres conceptualisations modernes (économie, art, droit, ...) une manifestation particulière : à ce propos, McCutcheon remarque à juste titre que « Like all the sciences, the history of religions is inextricably entrenched in a historical and theoretical framework and, therefore, it answers only the questions it poses, having little recourse to defending only its questions as normative $»^{17}$.

Dans un premier bilan, il me semble donc que la théorie de la « rupture de niveau » telle que Mora la propose n'est qu'un retour - à peine déguisé - aux anciennes thèses sur le "sacré » et les processus « empathiques » qui permettraient de s'approcher de la dimension religieuse, selon l'école phénomenologique et ses représentants majeurs, de R. Otto à G. van der Leeuw. Mora se rallie néanmoins explicitement à U. Bianchi et, plus généralement, à M. Eliade. Par conséquent, il ne me semble pas superflu d'approfondir les dettes que Mora admet avoir envers ces savants et d'examiner en quoi elles consistent.

L'expression « rupture de niveau » remonte en effet à $M$. Eliade qui, dans un passage de son Traité à propos des théories de Lokācharyā, affirme entre autres que " cette coïncidence sacré-profane réalise en fait une rupture de niveau ontologique. Elle est impliquée dans n'importe quelle hiérophanie, parce que toute hiérophanie montre, manifeste la coexistence des deux essences opposées : sacré et profane, esprit et matière, éternel et non éternel, etc. ${ }^{18}$.

En reprenant cette notion d'Eliade, Bianchi l'a reformulée mutatis mutandis dans de nombreuses études. Précisément concernant le passage cité, Bianchi a remarqué qu'il s'agit d'un concept qui « corrisponde a un'analisi positiva e scientifica dei fatti religiosi molto meglio di quelli vagamente postulatorii di 'ierofania', di 'simbolo', di 'irruzione' o 'rivelazione del sacro'; è meglio anche di quel concern with ultimate reality che è improptio per varii tipi di esperienza religiosa, non in tutto così ordinati alle prime e più solenni radici dell'essere, come lo sono, e in toto, le dottrine ufficiali delle grandi religioni ${ }^{19}$. Sans pouvoir approfondir ici ce problème, il faut néanmoins rappeler que pour Bianchi « la religione implica 'una rottura di livello' (...) e un primo aspetto del quid religioso può riconoscersi nel riferimento variamente e intensamente

Eliade 1953, p. 38.

19 Bianchi 1970 , p. 160. 
vissuto a un sopra-umano o a un extra-umano inteso come condizionante l'esistenza medesima del mondo e nel mondo ${ }^{20}$.

Du point de vue proprement historico-religieux, la notion de « rupture de niveau » - conçue comme la nécessité humaine de fonder, expliquer et/ou maîtriser une réalité qui échappe à tout contrôle technique et l'ancrer à des interlocuteurs extra-humains - pourrait être acceptée et se réveler même utile à la condition - comme Bianchi luimême l'a souligné - de n'être pas réduite à un vague ultimate concern, mais employée comme outil heuristique hors de toute définition essentialiste ${ }^{21}$. Il est pourtant tout à fait différent d'utiliser ce concept en posant comme hypothèse - comme Mora le fait - qu'il s'agit d'une "rupture de niveau " par rapport à la totalité d'une culture. L'on attribue de la sorte une dimension métaphysique invraisemblable à un outil de recherche : ceci implique la mise en jeu de jugements de valeur et de vérité et nous amène tout simplement au-delà et en dehors de l'histoire dont l'objet d'étude abstration faite des diverses options méthodologiques - ne peut être que la culture humaine dans toutes ses manifestations. En d'autres termes, la notion de "rupture de niveau » sera la bienvenue si elle nous aide à mieux comprendre la dimension spécifique de la religion à l'intérieur de la culture, tandis qu'elle est superflue ou carrément dangereuse, si on prétend définir par elle une autre dimension, radicalement différente du domaine de l'histoire.

En ce qui concerne Bianchi, même si lui aussi proposait de faire coïncider une définition minimaliste de religion avec la notion de "rupture de niveau », il a essayé de toute façon de justifier cette position en insistant toujours sur les fondements historiques qui devraient être à la base de cette démarche. Quant à Mora, il introduit ici les thèmes et les considérations de Bianchi d'une façon trop rapide et incomplète, de telle sorte que les prémisses historiques que Bianchi invoque toujours pour sa notion de "rupture de niveau » finissent par se dénaturer dans une nouvelle formulation, réduite et réductive.

Dans le paragraphe intitulé « La tipologia storica delle religioni ed il rapporto con le fonti » (que j'examinerai avec le paragraphe suivant, « Le religioni classiche nell'ambito della Storia delle religioni »), Mora se rallie de nouveau à Bianchi et en particulier à sa notion de «typologie historique» ("tipologia storica »), élaborée par

20 Bianchi 1991, p. 17. Ailleurs Bianchi explique sa pensée en remarquant que « Un primo aspetto del quid religioso possa riconoscersi nell'instaurazione di un rapporto di natura non visibile, per quanto talora sperimentabile, con un supra ed un pritus concepiti come condizionanti l'esistenza medesima del mondo. Il supra concerne l'essere o gli esseri che vengono concepiti come trascendenti, o comunque come superiori all'uomo quanto a potenza e gerarchia » (Bianchi 1986, p. 122). Encore : «Altrettanto si deve dire per gli atti e le cerimonie religiose; anche queste realizzano una 'rottura di livello' rispetto ai comportamenti profani, che le rende inassimilabili a questi, anche se non sempre agevole sarà, soprattutto presso certe religioni meno conosciute, il rendersi conto del limite esatto tra i due ordini di comportamenti. Il quale limite, d'altronde, male si valuterebbe ignorando il fatto parimenti essenziale che, anche qui, la distinzione è, secondo una celebre espressione, 'per unire' » (lbid., p. 123). Finalement : "Perciò la 'rottura di livello', la separazione dal profano, non realizza comportamenti stagni nell'uomo, ma una superiore unità: l'unione con il supra e con il pritus, quell'unione e armonia, quella pax deorum, alla quale in vari modi aspira, se vogliamo usare un termine di sapore cristiano, la condizione 'creaturale' dell'uomo » (ibid., p. 124).

21 Tout ceci semble être démenti par l'adjectif « ontologique » utilisé consciemment par Eliade dans le passage du Traité qu'on vient de citer (supra, n. 18). 
ce dernier « in ragione della rinuncia ad una definizione preliminare di religione ${ }^{22} \mathrm{e}$ della tensione ad una sempre maggiore approssimazione del concetto di religione attraverso la ricerca storica » (p. 13). D'après cette théorie, la recherche historique semble être le moyen pour atteindre non pas une connaissance des religions particulières ou de leurs éléments mais, au contraire, le concept général de religion, d'où précisément la nécessité d'élaborer une «typologie historique » visant à découvrir la continuité et les contrastes dans un domaine culturel « ben più vasto della singola civiltà ma ancora relativamente omogeneo ». Cette démarche permettrait, selon lauteur, d'éviter les «astratte comparazioni puramente fenomenologiche, tra usi e pratiche staccati dal proprio contesto (...) in un processo che riposa in larga misura su un'implicita ontologia della religione, quale sistematica organizzazione dell'esperienza religiosa, che dell'aprioristica definizione preliminare della religione sviluppa le premesse o l'intuizione originaria » (p. 13). Le concept de typologie historique, selon Mora, serait le seul qui puisse faire face à l'« esplosione quantitativa dell'oggetto degli studi storico-religiosi » et éviter que « l'impossibilità pratica di dominare l'insieme delle religioni porti alla dissoluzione dell'oggetto storico-religioso, quale prodotto di un'indagine non solo idiografica ma anche ed in primo luogo comparativa » (ibid.).

On affirme ici - sans ménager les termes - que l'histoire des religions, pour être une discipline historique et en même temps utiliser la méthode comparative sans tomber de nouveau dans la phénoménologie, doit avoir recours à la «typologie historique ». Il me semble que l'on arrive de la sorte à superposer deux ordres de problèmes. D'une part, la comparaison ne devrait pas dépasser la dimension culturelle caractéristique du phénomène à analyser (et dans le domaine duquel elle doit être contextualisée); d'autre part, elle devrait nous diriger vers la définition d'un concept de religion plus vaste, voire universel. La recherche devrait se diriger de la sorte vers « un ragionevole equilibrio tra l'ambito ristretto delle competenze individuali, di attiva ricerca storico-filologica, del singolo studioso e l'irrinunciabile esigenza che ogni indagine storico-religiosa resti aperta e rifluisca nella più ampia comparazione storicoreligiosa, volta all'elaborazione, graduale e paziente di un concetto storico di religione » (p. 13). Ici j'invite à réfléchir sur le fait qu'aucune discipline n'oblige ses spécialistes à maîtriser tout le savoir humain, même s'il est vrai que le « ambito ristretto delle competenze » du chercheur ne peut pas conditionner les horizons de la recherche: bien au contraire, toute enquête scientifique digne de ce nom impose au chercheur un élargissement adéquat de ses compétences et de ses outils au fur et à mesure que cela s'avère indispensable.

L'élaboration de «typologies » fondées sur la détermination de «types religieux » déduits de l'histoire est présentée finalement comme le choix stratégique qui s'impose pour l'enquête comparative en histoire des religions. Il n'est pas possible (et, du reste, il ne rentre pas dans mes objectifs) d'approfondir ici les énormes problèmes - aussi bien d'ordre théorique que pratique - soulevés par cette prise de position. Je désire tout simplement attirer l'attention sur le danger que n'importe quelle méthode puisse se transformer d'outil de recherche - plus ou moins performant - en objectif méta-bistorique de la recherche elle-même. La stratégie indiquée par Mora et inspirée de la pensée de Bianchi n'est qu'une parmi les différentes options méthodologiques possibles afin d'élaborer une taxonomie systématique des matériaux qu'on appelle

22 Mais cf. l'importance attribuée immédiatement auparavant à la notion de « rupture de niveau », invoquée comme critère pour clistinguer les faits religieux des faits non-religieux. 
conventionnellement « religieux». On pourra y adhérer ou pas, mais il n'est pas question de la considérer comme le but ultime de la recherche.

La méthode «typologique » comme telle n'a aucune connotation idéologique, elle est «neutre », pour ainsi dire. Il s'agit indiscutablement d'un processus classificatoire abstrait visant à déterminer et à élaborer des «types », eux aussi abstraits, en dépit du fait d'être déduits de la réalité historique. Sa valeur comme méthode dépend évidemment de l'usage qu'on en fait et des résultats qu'on obtient. Certes, comme toute méthode qui aspire à une dignité scientifique - même dans les limites imposées par le caractère des sciences humaines (c'est une vieille question!) - elle doit être conçue et élaborée avec la plus grande rigueur et doit en plus posséder un degré considérable d'élasticité pous s'adapter aux objectifs posés chaque fois par la recherche historique. Si ce n'est pas le cas, on court un risque majeur car, pour rappeler les mots de H. Biezais, on constate « daß sich ohne strikte Definition des Typusbegriffs unüberwindliche Schwierigkeiten bei dessen methodischer Anwendung ergeben ${ }^{23}$.

Que l'on ait affaire à des problèmes d'une complexité extraordinaire est confirmé par le vieux débat scientifique concernant la « comparabilité » des religions, à l'intérieur duquel rentre aussi la question des praxis méthodologiques à adopter. Il est clair que les concepts de «type », «modèle », « catégorie», « classe » etc. sont toujours (plus ou moins explicitement) employés par les spécialistes, mais ils restent tous des outils heuristiques et non pas des entités abstraites pourvues d'une validité universelle. Toute prétention de leur attribuer une telle portée - il vaut mieux le répéter - est dépourvue de fondement. Nos schémas classificatoires sont des élaborations subjectives, ils impliquent des processus de réduction individuels et ne peuvent pas posséder une validité absolue. Comme Biezais l'a justement remarqué, il faut être bien conscient du fait « daß der Typus deshalb, weil ihm die Qualität einer absoluten Kategorie fehlt, nur von relativer Bedeutung ist. Diese kann auch durch einen intersubjektiven Sachverständigen-Consensus nich erhöt werden ${ }^{24}$.

Il est tout à fait possible que deux (ou plusieurs) chercheurs, engagés dans la détermination de «types » et dans l'élaboration de «typologies » tirées de l'étude des faits historiques, arrivent à des conclusions qui sont très différentes l'une de l'autre. Ceci dépend du caractère subjectif de leurs choix, conséquence de leur formation scientifique et de leur's intérêts.

Tout ceci ne signifie pas, bien sûr, que le savant doive renoncer à travailler en utilisant des concepts et des catégories abstraites (prétention par ailleurs impossible), mais c'est tout autre chose d'affirmer que ces concepts et ces typologies possèdent une validité objective, universelle et éternelle. La prétention de s'en servir pour déterminer un concept historique de religion toujours valable - dans celle qui pour Mora est la phase décisive et finale de l'enquête historico-religieuse - paraît plus que douteuse : dans la meilleure des hypothèses, elle implique un processus de réduction de dimensions colossales et susceptible de nous réserver bien des surprises. À ce sujet, Ph. Borgeaud observe judicieusement : « Le plus souvent, en effet, l'historien des religions est l'homme d'un terrain. Les questions comparatistes qu'il se pose sont dictées par les conditions mêmes de son terrain. Cela, qui est le plus intéressant, n'exclut pas la légitimité d'une enquête qui se donnerait pour but une classification générale des religions, mais entraîne comme conséquence que les critères d'une telle entreprise seront toujours arbitraires, et partiaux. Une telle classification ne peut s'effectuer que

23 Biezais 1981, p. 19.

24 Biezais 1981, p. 15. 
sous un certain angle, et en réponse à certaines questions (...). Toute classification générale, par conséquent, aurait le statut d'un essai, avec ce que cela représente de stimulant mais aussi d'inachevé (...) la comparaison n'a plus pour but (sinon ultime dans le sens d'inacessibile) une classification générale (...) $\gg^{25}$. Encore avant Borgeaud, toutefois, A. Brelich écrivait que l'histoire des religions est « in teoria (...) la ricerca fondata sulla comparazione, della storia di tutte le formazioni religiose del passato e del presente, che già si conoscano. 'In teoria', si è detto, perché ovviamente un siffatto fine non può essere perseguito da singoli studiosi (...); perciò, nella pratica, essa è soprattutto un metodo, la cui assimilazione, accompagnata da una preparazione quanto più larga, mette in grado lo studioso di comprendere storicamente i singoli fatti religiosi di qualsiasi epoca o civiltà, per la conoscenza della quale acquisisce gli strumenti (filologici, archeologici, storico-politici, ecc.) necessari ${ }^{26}$.

En tout cas la méthode "typologique " - abstration faite de son utilité - n'est jamais susceptible de nous faire découvrir des qualités de signification universelle, puisque son élaboration se fonde sur une «subjektive Auswahl (scil., de la part du chercheur) der Eigentümlichkeiten (...), wobei die Auswahl sich nach dem Forschungsziel richtet ${ }^{27}$. La substance des faits ne change nullement si l'on ajoute l'adjectif " historique " : une typologie - on vient de le remarquer - est toujours abstraite par définition, même si elle se fonde sur l'observation des phénomènes historiques. Une fois reconnue l'utilité d'une telle méthode, il faut toujours se souvenir qu'elle est possible " nur als eine Konstruktion (...), um sich in der Mannigfaltigkeit der Wirklichkeit zu orientieren $»^{28}$.

En revenant aux arguments de Mora, la comparaison en histoire des religions servirait à évaluer le « carattere religioso dei fatti documentati, la loro interpretazione e l'individuazione di tipologie storiche ». Rien qu'à elle «spetta la ricostruzione del fenomeno e la sua classificazione in relazione ai fenomeni comparabili ». Mais Mora ajoute aussitôt que la recherche doit se nourrir " di continua attenzione al carattere storico dei fatti studiati, mediante il loro accertamento puntuale, idiografico ». Du point de vue pratique, «spetta alla sensibilità dello storico delle religioni porre domande motivate dalle conoscenze storico-comparative, ma l'accertamento delle risposte dipende dal corretto uso delle metodologie storiche necessarie a verificare fatti specifici nelle civiltà in questione ».

Pourquoi, pourrait-on se demander, les questions posées par le chercheur doivent-elles être formulées d'après une « sensibilité » générique ? Comment peut-on vérifier s'il existe une telle attitude? Pourquoi, encore, la fonction de vérification garantie par une méthode historique n'entre-t-elle en jeu que dans l'évaluation des réponses aux questions formulées sur la base de l'intuition? Est-il concevable que le

25 Borgeaud 1986, p. 68.

26 Brelich 1979, p. 255.

27 Biezais 1981, p. 23.

28 Ibid. Last but not least, une dernière question se pose spontanément, qui peut être formulée de la manière suivante : quels sont les rapports entre ce résultat final qui pourrait mettre fin à l'histoire des religions (à savoir, le concept de religion atteint grâce à l'élaboration d'une typologie complexe), et l'intuition du départ, c'est-à-dire, le concept de religion expérimenté de façon subjective? On se demande si ce n'est pas ce dernier le plus riche, le plus vrai, le plus fondé du point de vue historique par rapport au concept « universel ». Ce concept « universel », par ailleurs, même s'il est déduit des faits historiques, est le résultat d'une gigantesque réduction fondée sur l'élaboration (toujours subjective, bien sûr) de microtypologies et macrotypologies, élaboration qui se fonde à son tour sur la détermination et la classification de très nombreux types. 
choix et la formulation des questions puissent s'effectuer sans l'aide et le contrôle de la même méthode invoquée dans la phase de vérification des résultats obtenus ? Il suffit de lire attentivement les pages de Mora pour se rendre compte des apories considérables qui caractérisent ici sa refléxion.

J'estime en réalité que toutes les phases de l'enquête - pourvu qu'on arrive à les distinguer aussi nettement - doivent être soumises mutatis mutandis à l'utilisation consciente de la même méthode. Qu'il s'agisse de deux côtés d'une même médaille est confirmé par le fait que la documentation elle-même conditionne et oriente le chercheur dans une direction ou dans l'autre, aussi bien dans le choix des problèmes que dans la formulation des questions. Le chercheur est un sujet unitaire qui ne doit pas souffrir de schyzophrénie : il réfléchit, il se pose des questions, il cherche les réponses, il soumet le tout à vérification en employant une méthode unitaire qui, dans le cas de l'histoire des religions, devrait conjuguer harmonieusement histoire, comparaison et philologie dans le sens le plus complet du terme ${ }^{29}$.

Selon Mora, encore, « il metodo storico si pone come l'ausiliario del metodo storico-comparativo ». Ici aussi je dois remarquer que c'est plutôt le contraire qui est vrai : la méthode historico-comparative est une spécialisation de la méthode historique générale qu'on applique à un ensemble de phénomènes appartenant à des contextes culturels particuliers : ils ne peuvent pas être définis comme « religieux » a fortiori, mais ils se signalent comme tels précisément grâce à la comparaison avec des phénomènes semblables du point de vue morphologique et fonctionnel. En ce qui concerne l'indispensable phase idiographique de l'enquête - sans laquelle on ne peut parler ni d'objet ni de méthode - c'est à mon sens précisément la capacité d'élaborer des dossiers précis et rigoureux concernant des systèmes particuliers qui rend possible une comparaison également rigoureuse et attentive.

L'auteur en arrive alors à souligner le conditionnement exercé par les sources dans les études d'histoire des religions. Il en ressort ce que Mora lui-même appelle une « sommaria tipologia » delle civiltà, volta a distinguerle in base al carattere delle fonti disponibili (scrittura/oralità, fonti primarie e/o secondarie, etc.). Cette typologie est jugée comme particulièrement adaptée pour déterminer « $\mathrm{i}$ problemi di indagine storica posti dalle religioni del mondo classico e dei popoli limitrofi » (p. 15).

On atteint là le cour de l'argumentation. Mora adopte une définition « élargie » de monde classique en y incluant « tutte le civiltà dotate di scrittura note agli autori greci e latini, in aree poi oggetto di penetrazione greca o latina, da Omero all'espansione araba » et y incluant « non solo Greci e Latini, ma anche Etruschi, Italici, Egizi, Fenici, Ebrei, altri popoli semitici, civiltà mesopotamiche, micrasiatiche, Iran » (p. 1516), toutes civilisations considérées comme appartenant « a pieno titolo alla koine culturale ellenistico - romana, almeno in una certa fase della loro storia ${ }^{30}$. Cette classification - fondamentalement centrée sur l'époque hellénistique et romaine correspond manifestement aux intérêts personnels de Mora et se révèle ipso facto arbitraire et inadéquate à représenter la diversité et la complexité des cultures prises en considération. En effet, Mora apporte tout de suite une correction de perspective

29 «The special character of the history of religions (...) lies in its combination of philology, history, and comparison », Rudolph $1985 \mathrm{a}$, p. 105.

30 L'on remarquera que pour de nombreuses cultures extra-classiques cette « certa fase della loro storia » n'est qu'un minuscule segment de leur histoire. À Mora revient entre autres le fardeau de la preuve lorsqu'il s'agit d'expliquer l'importance attribuée aux sources gréco-latines pour la connaissance globale des religions comme les religions sumérienne, élamite ou hittite (etc.). 
apparemment marginale, mais en réalité substantielle, car il modifie ses critères géographiques et chronologiques en y introduisant le concept de « Alte culture del mondo antico ", en plus de l'inévitable récupération de ceux qu'il définit " peuples limitrophes ». Le résultat final n'est qu'une juxtaposition assez confuse et fragmentée de civilisations que les remarques qui suivent - visant à conférer un minimum d'ordre au tableau esquissé - n'arrivent pas à rendre plus cohérente.

Pour ne proposer qu'un exemple, pour Mora les Égyptiens ne sont pas «limitrophes » puisqu'ils possèdent des sources écrites, mais leur civilisation se trouve partagée en deux blocs : toute la période, disons, pré-homérique (point de repère tout à fait étranger à leur histoire !) les voit classés parmi les « hautes cultures du monde antique », tandis qu'à l'époque hellénistique et romaine ils sont « récupérés »apparemment comme civilisation de second rang - au moins sur la base de la documentation gréco-latine, considérée comme le point de repère capital. Pourquoi se refuse-t-on à considérer la culture (et la religion) égyptiennes - même dans leur dynamisme historique - comme unitaires et dignes d'une approche exhaustive menée à travers les sources égyptiennes elles-mêmes? Et le concept de " peuples limitrophes » appliqué sur la base d'un manque de sources écrites? Est-il légitime de fonder le critère d'existence autonome d'une culture sur la connaissance éventuelle de cette dernière par les écrivains grecs et latins ? Pourquoi le centre de gravité chronologique d'une classification «typologique » des religions anciennes doit-il être forcément l'époque hellénistique et romaine? Que faire alors des peuples « limitrophes des limitrophes »? Le spécialiste de la religion mésopotamienne ou, par ex., de la religion celtique, ne seraient-ils pas attitrés à orienter cette classification de leur point de vue? Et encore : pourquoi ne peut-on pas étudier chaque civilisation dans une perspective diachronique en tenant compte que, selon les époques, on a affaire à des sources différentes et à une certaine période il nous est possible d'utiliser aussi les informations des auteurs grecs et latins? L'importance attribuée à ces derniers et aux civilisations respectives ne dérive-t-elle pas d'une vision « classicocentrique » si naïve qu'elle ne pense même pas à se justifier ${ }^{31}$ ? A-t-on vraiment besoin de typologies qui violent l'histoire et compliquent démesurément la tâche déjà ardue des spécialistes des religions du monde antique?

La plupart de ces questions - qui attendent des réponses adéquates et satisfaisantes - sont suscitées par les critères singuliers choisis par Mora : une distinction entre sources primaires et sources secondaires, l'importance démesurée attribuée à ces dernières et le rôle capital reconnu aux sources en langue grecque et latine. Il serait trop compliqué de résumer ici dans les détails toutes les considérations de Mora, et en particulier son articulation des sources primaires et des sources secondaires. Il faut seulement rappeler que les sources primaires sont pour lui les documents directs (par ex., les textes cunéiformes, les documents en sanscrit, etc.), les sources secondaires sont les témoignages indirects (« riflesse ») produits par les tenants de telle ou telle culture, et qui fournissent une représentation des faits

31 Cf. par ex. ce que Mora affirme dans sa réflexion sur les sources : « Delle religioni del 'mondo classico', nel senso più ampio del termine (tale cioè da comprendere anche le 'Alte culture del mondo antico' e i 'Popoli limitrofi'), due, e cioè quella greca (d'epoca classica ed ellenistica) e quella romana (dalle guerre puniche a tutta l'epoca imperiale) si prestano in modo particolare alla ricerca moderna (...) per la presenza di fonti monumentali e scritte, che comprendono anche testimonianze riflesse, e ancor più per l'utile presenza di fonti incrociate » (p. 19). Je ne pense pas que les spécialistes des religions extra-classiques (il suffit de songer à l'Islam ou au Judaïsme) souscriraient volontiers à ces affirmations. 
religieux plus « proche » d'eux. Mais les sources « primaires » prises en considération dans le cadre d'une étude historique - il s'agit très souvent, à ma connaissance, de la plupart des documents - demandent aussi d'être évaluées critiquement puisque ellesmêmes sont sujettes à des conditionnements idéologiques divers : le problème est alor's de déterminer le niveau d'intervention afin d'y apporter les décodages nécessaires.

À ce propos, Mora écrit : « le fonti primarie, specie quelle non ampie e soprattutto non mitiche ${ }^{32}$, spesso non sembrano presentare allo studioso sufficienti elementi per un'interpretazione autonoma, cioè prevalentemente fondata sull'informazione che contengono, che peraltro in molti casi può essere raggiunta con un'analisi sistematica della documentazione primaria ». Tout ceci s'avère vrai en tout cas, surtout lorsque nous avons affaire à des témoignages du culte et non pas à des «traités " redigés par les membres d'une culture «autre » et concernant leurs propres croyances. Aucune reconstruction historique ne peut être entamée en partant d'un seul type de source et sans le contrôle et l'évaluation comparative avec d'autres documents issus de la même culture : en d'autres termes, aucune information ne peut être considérée comme « autonome».

Dans ce contexte, il est tout à fait surprenant (et quelque peu contradictoire) que Mora arrive à denoncer le « peso tuttora prevalente dell'interpretazione greco-romana e delle equivalenze stabilite dagli autori classici nella comprensione delle strutture religiose degli altri popoli del mondo classico (...), anche di quelli dotati di un'ingente documentazione scritta ». Cette situation reflète à mon avis une tendance perceptible dans les études d'histoire des religions selon certaines traditions universitaires, où il y a une sorte de discrimination implicite entre Altertumswissenschaftler d'une part, et spécialistes d'autres civilisations (orientalistes : Proche- et Extrême Orient, américanistes, etc.) d'autre part. Tandis que les premiers sont presque automatiquement reconnus comme historiens des religions (c'est le cas de l'Italie en particulier), les savants engagés dans l'étude d'autres cultures et religions sont généralement considérés comme des philologues. Raisons d'ordre académique mises à part - il faudrait ici une analyse socio-culturelle d'une tout autre orientation - il faut se demander si, dans le cas qui nous concerne, cette lecture en clé classico-centrique des civilisations «autres» (« hautes cultures du monde antique», peuples limitrophes et limitrophes des limitrophes) ne s'explique pas par la difficulté des spécialistes du monde classique à maîtriser les «sources primaires » (dans le sens de Mora, cf. supra), avec le résultat de privilégier les documents auxquels ils peuvent avoir un accès direct.

De toute façon, le discours de Mora trahit la tendance à présenter comme des nouveautés absolues des questions débattues depuis longtemps et auxquelles, dans plusieurs cas, on est arrivé à fournir des réponses satisfaisantes, bien que provisoires. Par ex., là où il affirme que « La fonte secondaria può appartenere ad una cultura diversa da quella su cui riferisce : in questo caso si apre una problematica di grande interesse e solitamente trascurata, e cioè un'analisi delle testimonianze etnograficoreligiose volta non a studiare le civiltà testimoniate, ma a capire gli schemi che sorreggono la rappresentazione della civiltà e dei suoi elementi nella cultura dello storico (antico), anche alla luce della frequente differenziazione, in gradi diversi, tra cultura propria, affine, 'altra' o anche non-cultura » (p. 18). Il est tout à fait surprenant

32 Il vaut la peine de rappeler que l'histoire des études connaît de nombreuses analyses et décodages de mythes qui représentent des contributions fondamentales pour la connaissance des religions auxquelles ils appartiennent. 
que dans ce cas aussi Mora ne trouve rien de mieux que de se citer lui-même, faisant semblant (?) d'ignorer que le décodage indispensable des sources a été largement théorisé et expérimenté, par ex. en Italie, par divers spécialistes appartenant à la soidisant «Scuola di Roma ». Le cas des noms propres théophores, cité par Mora, n'est qu'une goutte dans l'océan de cette vaste problématique. Un spécialiste du monde classique aurait dû mentionner (entre autres) au moins les cas de Manéthon, Bérose et Philon de Byblos; mais, plus encore, il aurait fallu faire allusion en général aux recherches menées sur les processus de «traduction» d'une culture à l'autre, sur les mécanismes d'acculturation, sur les conditionnements dont le chercheur moderne doit toujours tenir compte - une fois qu'ils ont été déterminés - dans ses analyses des phénomènes d'une culture, y compris celles que nous appelons d'une manière conventionnelle « religieux».

Parmi le résultats obtenu par l'analyse critique des sources (en particulier, celles que Mora appelle « croisées », [ incrociate »]), il y aurait, selon l'auteur, la possibilité « di approfondire in misura ancora maggiore la soggettiva percezione della religione, fino a tratteggiare, per i singoli autori antichi, una tipologia della Religione in generale, dei suoi elementi costitutivi e del rapporto tra la Religione in sé [c'est moi qui souligne] e le sue varianti costituite dalle singole religioni » (p. 18-19). Si j'ai bien compris, il s'agirait de saisir derrière les témoignages anciens l'idée de religion en soi que chaque auteur doit posséder. Dans les termes qu'il emploie, cette affirmation est trop vague : comment peut-on parler en général de «autori antichi »? Les documentations dont on dispose aujourd'hui sont très variées, les raisons pour lesquelles certains individus ont été poussés à réfléchir sur leur propre religion ou sur d'autres religions (soit prises en général, soit, plus souvent, sur des aspects spécifiques) sont très différentes et sont liées à des prémisses tout à fait diverses : la « religion » (là où ce concept existe) n'emerge pas comme quelque chose en soi, dont les religions particulières seraient de simples variantes. C'est une chose que de parler d'Hérodote, une autre chose que de se référer aux hérésiologues ou aux apologètes des premiers siècles de notre ère qui malgré tout appartiennent tous, selon la précision de Mora, au «monde classique ». Le concept de « religion ${ }^{33}$, il est peut-être superflu de le rappeler, «si è formato (...) nel corso della storia della civiltà occidentale. E' essenziale ribadire - se pure ve ne fosse il bisogno - che nessuna lingua dei popoli primitivi, nessuna civiltà, nessuna civiltà superiore arcaica, neppure gli stessi greci e i latini più vicini a noi dispongono di un termine corrispondente a questo concetto, che è andato definendosi storicamente in una certa epoca e in un determinato ambiente, con ulteriori autonomi sviluppi ${ }^{34}$.

Par conséquent, il me semble impossible d'enquêter sur les auteurs anciens en parlant de "perception subjective de religion ", mais il faut plutôt aller à la recherche d'attitudes individuelles face à des faits, des traditions ou des croyances perçus comme différents des siens propres. Un problème dans le problème est en outre représenté par les apports individuels, souvent oubliés mais parfois surévalués, sans compter que pour les cultures du monde antique le document est le plus souvent anonyme, et toute la question doit être abordée sur des bases différentes. Cette problématique, du reste - il le savent bien les anthropologues et les ethnologues, si souvent négligés par les spécialistes du monde classique - n'est pas du tout limitée

33 C'est autre chose que la «Religione in sé » dont Mora parle, qui n'existe pas hors de l'histoire et, par conséquent, ne peut pas rentrer pas dans les objectifs de l'histoire des religions.

34 Brelich 1979, p. 140. Sur « religion » voir en général Feil 1986. 
aux « faits religieux», mais elle concerne à plein titre tous les aspects des cultures mises en cause et étudiées d'une manière comparative.

Un mot encore doit être prononcé sur le panorama fourni par Mora des autres « religions classiques ». Je ne me considère absolument pas comme une fanatique des longues bibliographies, et encore moins de l'exhaustivité à tout prix. Mais je reste quand même étonnée en constatant que la source utilisée par Mora sur les «autres » religions est presque seulement un travail collectif de «Storia delle religioni » (éd. Castellani) qui va avoir désormais trente ans ${ }^{35}$. Les rares exceptions sont elles aussi étonnantes. Comme source fondamentale pour la religion hébräque, p. ex., on mentionne la syntèse bien dépassée de H. Ringgren ${ }^{36}$. En me limitant à une seule illustration à cet égard, il aurait fallu mentionner au moins les deux volumes de R. Albertz, auteur d'un satisfaisant status quaestionis sur l'ancien Israël ${ }^{37}$. La bibliographie sur les religions de la Mésopotamie est elle aussi largement insuffisante, limitée aux contributions contenues toujours dans Castellani (articles de G.R. Castellino et de L. Cagni). À ce propos, chacun sait que le regretté L. Cagni, auteur d'une section très vaste de ce travail, l'avait complètement réélaborée pour la nouvelle édition de l'ouvrage de Castellani, cette fois éditée par G. Filoramo, avec une mise à jour radicale de son texte ${ }^{38}$. Pourquoi alors ne pas citer le dernier Cagni, si on a confiance en cet auteur? Encore, G. Furlani a été sans aucun doute un savant de premier plan dans l'étude des cultures cunéiformes mais, certes, il n'était pas spécialement hittitologue, de sorte que la mention de son ancienne contribution (et en particulier de sa malheureuse affirmation que le panthéon hittite « ci è noto solo nella tradizione 'scrittoria' sumera »), finit par discréditer complètement l'apparat bibliographique concernant l'Anatolie. En omettant d'insister sur d'autres cas dignes pourtant d'être remarqués (comme par ex. celui de la religion étrusque), le traitement réservé à la Syrie ancienne est particulièrement éclatant. Ici on mentionne une contribution de G. Garbini antérieure à la découverte d'Ebla, un événement qui a révolutionné nos connaissances sur les cultures et les religions de l'aire syro-palestinienne. La « grande limite » ("grosso limite ») des religions syriennes anciennes repérée par Mora consisterait dans la «presenza di insufficienti testimonianze riflesse »: or, cette limite n'a certes pas empêché la recherche historico-religieuse dans ce domaine ${ }^{39}$, bien sûr conditionnée - mais non pas paralysée - par le type de sources dont on dispose. Tout cela équivaut à affirmer que, par ex., l'étude de la religion romaine serait limitée par l'absence d'une mythologie de type traditionnel, où que l'étude de la religion grecque serait dérangée par l'absence de textes rituels comme ceux d'Ugarit, ou bien par le manque d'un texte sacré comme le Coran.

Ici on ne se trouve pas sur le plan de la recherche idiographique, mais tout simplement sur celui de la mise à jour bibliographique, particulièrement indispensable lorsqu'on est obligé d'utiliser des sources qu'on n'arrive pas à maîtriser directement et, surtout, lorsqu'on on invoque l'attention internationale pour des propositions importantes comme une nouvelle typologie des religions du monde antique.

Si on en vient finalement à approfondir dans les détails cette « typologie » des religions classiques élaborée par Mora (p. 21 sq.), on tombe sur un paragraphe intitulé

35 Castellani 1970.

36 Ringgren 1981 (à son tour, réédition d’un original encore plus ancien).

37 Albertz 1992.

38 Cagni 1994.

39 Cf. par ex. Xella 1994; Niehr 1998. 
«Elementi di una tipologia delle religioni classiche », où en réalité Mora ne s'occupe que d'un seul " élément » typologique, à savoir le polythéisme. Ce terme est introduit par l'auteur en renvoyant à un de ses articles ${ }^{40}$, sans qu'il juge opportun d'en donner au préalable aucune notion d'orientation, d'autant plus qu'il s'agit d'un thème très controversé précisément à cause de la présence de l'élément -théisme, qui réclame une approche rigoureuse aussi bien du point de vue historique que de la définition ${ }^{41}$.

Selon notre auteur, les aspects fondamentaux du polythéisme sont « l'organizzazione sistematica non geografica delle figure divine, la replicabilità in luoghi diversi di questa organizzazione e il carattere astratto ed universale delle figure divine (che presuppongono forme differenziate di rapporto coi fedeli), accompagnate, quale elemento stabilizzatore, da una relativa laicizzazione, sovente congiunta ad una tematica classificatoria $\gg\left(\right.$ p. 22-23) ${ }^{42}$. Le point de repère absolu est le polythéisme grec, qui représente « una forma assai marcata, compiuta di questa struttura religiosa $\gg{ }^{43}$. À ce dernier, Mora attribue une ouverture universaliste particulière, à savoir une volonté de relecture des expériences religieuses « autres » à l'intérieur de son propre panthéon et selon son propre langage mythologique. Il s'agit ici - c'est presque superflu de le remarquer - d'une interprétation gréco-centrique qui néglige, entre autres, en renversant l'optique, d'aborder la question de savoir comment le panthéon et les dieux grecs pouvaient être assimilés par d'autres univers religieux avec lesquels ils sont venus en contact. À cet égard, l'absence de sources n'est pas une preuve contraire. Tout processus d'acculturation ne se déroule jamais à sens unique : il y a toujours différents niveaux de relecture et de réorganisation, particulièrement en l'absence d'une orthodoxie et de canons reconnus et appliqués.

J'éviterai de résumer ici les considérations de Mora sur une série d'aspects de la religion grecque, ainsi que la comparaison rapide qu'il établit avec la religion romaine. Peut-être à cause du caractère très synthétique de ces réflexions - le thème mériterait bien plus qu'une vaste monographie - son discours donne l'impression de se fonder sur des remarques éparpillées, où les différences et les analogies mises au jour dans les deux religions semblent tout à fait détachées de la structure générale des cultures respectives. Qu'il me soit permis de manifester des doutes sur certaines affirmations qui concernent par ex. le processus d'identification entre la religion grecque et la religion romaine (par ex., en condamnant la religion grecque, les apologistes chrétiens auraient pratiquement eu l'intention de soutenir la religion romaine, considérée comme plus adéquate à être comparée au christianisme); ou bien, sur la formulation des possibles «points de contact » entre religions différentes, juxtaposées l'une à

40 Mora 1994.

41 Sur ce problème cf. entre autres Brelich 1960; Detienne 1986; Schmidt 1987; Gladigow 1995 et 1997; Sabbatucci 1998.

42 Sans vouloir ici alourdir ma réfléxion, je me limite à remarquer que le critère de l'« organizzazione sistematica non geografica » des figures divines est démenti précisément au pays de Sumer où, comme désormais tout le monde s'accorde de reconnaître, on trouve la plus ancienne manifestation du polythéisme de l'histoire.

43 Ceci implique que pour Mora il y a des formes «inachevées " «incompiute ») de polythéisme et que l'on a affaire - en perspective phénoménologique - à une structure idéale et ahistorique dont il faut déterminer les formes historiques, qui seront ensuite évaluées d'après le degré de ressemblance au « modèle ». Au-délà du caractère tendanciel/ potentiel du polythéisme (un aspect bien éclairci par Brelich 1960), on retrouve ici en outre la tendance à utiliser les civilisations classiques comme point de répère privilégié, comme lieu idéal de réalisation des formes et des phénomènes particulièrement exemplaires. 
côté de l'autre phénoménologiquement, sans un intérêt spécifique pour les différences idélogiques et fonctionnelles, parfois plus marquées que les ressemblances. Est-il vraiment possible de comparer sic et simpliciter l'absence du mythe dans la religion romaine et dans la religion d'Israël ? Ou de mettre sur le même plan la pax: deorum romana et la berit des Hébreux? Sans compter que Mora mélange (confond ?) ici la religion hébraïque avec celle de la Bible : cette dernière - c'est une notion acceptée même par les Alttestamentler les plus fondamentalistes - est une construction à posteriori des âges exilique et postexilique et ne reflète qu'en partie (et seulement pour des époques tardives) la réalité religieuse du peuple d'Israël, beaucoup plus ouvert au polythéisme et aux cultes «sessualmente caratterizzati » (l'expression est de Mora) qu'on ne le pense d'habitude.

Après avoir constaté les difficultés qu'on rencontre dans l'étude des «antiche religioni 'primitive' », Mora mentionne, à côté de la perspective polythéiste grecque «quale religione nazionale, considerata omogenea nelle diverse repliche locali esperite come pienamente equivalenti dai singoli fedeli » (p. 26), la vision opposée, issue d'une perspective monothéiste qui « sviluppa schemi interpretativi non individuali, ma generici e applicati di volta in volta a divinità diverse, ed in particolare la figura del dio creatore » (p. 26). Ce type de polythéisme serait caractéristique de la religion phénicienne " quasi per atrofizzazione della componente classificatoria presente nella religione ugaritica » (p. 26). Il s'agit d'une affirmation surprenante qui ne pourra pas être partagée par les spécialistes de ce domaine, négligés par Mora dans sa bibliographie, limitée à une autocitation ${ }^{44}$.

À propos de la religion (= polythéisme) de l'Égypte, considérée en bloc en dépit des millénaires de son histoire, Mora s'exprime de nouveau en utilisant le concept de «manque » par rapport au polythéisme grec. La religion égyptienne manquerait, entre autres, de « un sistema classificatorio ad un tempo locale e nazionale », par conséquent le spécialiste est obligé d'avoir recours, comme seule clé d'interprétation (« principio individuante »), à la « combinazione di un criterio geografico e di un altro basato su tratti funzionali e ancora più teriomorfici » (p. 26). De nouveau, on met en évidence « un'anomala tendenza [c'est moi qui souligne] a combinare liberamente figure divine per realizzarne altre » due à la «mancata distinzione dipartimentale di funzioni e caratteristiche diverse » (ibid.). De toute façon, le polythéisme ne serait qu'une parmi les « grandezze fondamentali » à analyser afin de réaliser une «articolata tipologia delle religioni classiche » (p. 27). À la fin nous restons sur notre faim puisque Mora effleure à peine une série de thèmes et de problèmes primordiaux qui concernent surtout le monde hellénistique et celui de l'antiquité tardive, problèmes dont la formulation suit généralement la Weltanschaumng chrétienne et occidentale : c'est le cas, entre autres, de la «faute individuelle », de la sotériologie, de la protologie / eschatologie.

Dans le dernier paragraphe, «Paradigmi come strumento di analisi diacronica delle religioni classiche » Mora mentionne enfin la proposition - due à H. Küng d'appliquer aux sciences historiques le concept de paradigme tiré des sciences de la nature. Abstration faite des préférences individuelles en matière de terminologie, on n'entrevoit aucune perspective nouvelle dans cette approche, qui se clôt sur une tentative de déterminer une série de phases à l'intérieur de la religion grecque et romaine. 
Voici mes réflexions. Mora, il faut le répéter, a manifesté beaucoup de courage, mais sans doute ne s'est-il pas rendu compte des obstacles considérables disséminés sur son chemin et il a fini par entreprendre trop de choses à la fois. Condenser en quelques pages une reconsidération globale de l'objet et de la méthode en histoire des religions; élaborer une typologie fondée sur le rapport entre les civilisations et les sources disponibles; déterminer la portée et les limites de la notion de "religions classiques »; fournir les éléments d'une classification typologiques de ces dernières, en introduisant en plus le concept de « paradigme » : tout ceci dépasse largement les possibilités objectives d'un auteur dans l'espace d'un article. C'est une entreprise pourvu qu'on soit bien convaincu de son utilité - pour laquelle aurait été indispensable - dès le début - l'emploi prudent et rigoureux de termes-clés comme par ex. « religion », «type », « polythéisme », « mythe », etc.

On se voit par contre projeté brusquement dans un univers conceptuel où l'existence d'une expérience religieuse, d'une conduite religieuse, de faits religieux est considérée conme acquise, sans aucune explication préalable des critères qui autoriseraient à définir comme « religieuses » cette expérience et cette conduite, ou comme "religieux » ces faits. On se trouve ici confronté à des problèmes qu'il faut aborder avec lucidité et cohérence et dans le but de faciliter les études d'histoire des religions, non pas de les dérouter vers des objectifs improbables qui finissent par compliquer inutilement la recherche.

Maria Grazia LANCELLOTTI

Via Tigre, 33

I - 00199 ROMA

\section{Abréviations bibliographiques}

Albertz 1992 : R. Albertz, Religionsgescbicbte Israels in alttestamentlicher Zeit I-II, Göttingen, 1992.

Bauman 1993 : Z. Bauman, Postmodern Etbics, Oxford, 1993.

Bianchi 1970 : U. Bianchi, "La storia delle religioni", in Castellani, 1970, I, p. 1-168.

Bianchi 1975 : U. Bianchi, The History of Religions, Leiden, 1975.

Bianchi 1983 : U. Bianchi, "Il metodo della storia delle religioni", in A. Molinaro (éd.), Le metodologie della ricerca religiosa, Roma, 1983, p. 17-28.

Bianchi 1986 : U. Bianchi, Problemi di storia delle religioni, Roma, $1958\left(1986^{2}\right)$.

Bianchi 1991 : U. Bianchi, Saggi di melodologia della storia delle religioni, Roma, $1991^{2}$.

Bianchi 1994: U. Bianchi (éd.), The Notion of "Religion" in Comparative Research. Selected Proceedings of the XVIth Congress of the International Association for the History of Religions, Rome, 3rd-8tb September 1990, Roma, 1994.

Biezais 1981 : H. Biezais, "Die typologische Methode in der religionsgeschichtlichen Forschung", Temenos 17 (1981), p. 5-26.

Boespflug - Dunand 1997 : F. Boespflug, Fr. Dunand (éds.), Le comparatisme en bistoire des religions, Paris 1997.

Borgeaud 1986 : Ph. Borgeaud, "Le problème du comparatisme en histoire des religions", Revue européenne des sciences sociales 24 (1986), p. 59-75.

Braun - Henking 1999 : H.-J. Braun, K.H. Henking (éds), Homo religiosus, Zürich, 1999.

Brelich 1960 : A. Brelich, "Der Polytheismus", Numen 7 (1960), p. 123-136.

Brelich 1979 : A. Brelich, Storia delle religioni : percbé?, Napoli, 1979. 
Cagni 1994 : L. Cagni, "La religione della Mesopotamia", in G. Filoramo (éd.), Storia delle religioni I, Bari, 1994, p. 115-176,

Castellani 1970 : G. Castellani (éd.), Storía delle religioni (fondata da P. Tacchi Venturi), Torino, $1970^{6}$

Cox 1998 : J.L. Cox, "Religious Typologies and the Postmodern Critique", Method \& Theory in the Study of Religion 10 (1998), p. 244-262.

Detienne 1986 : M. Detienne, "Du polythéisme en général", CPb 81 (1986), p. 47-55.

Detienne 1997 : M. Detienne, "Expérimenter dans le champ des polythéismes", Kernos 10 (1997), p. 57-72.

Eliade 1953 : M. Eliade, Traité d'bistoire des religions, Paris, $1953^{2}$.

Feil 1986 : E. Feil, Religio. Geschichte eines neuzeitlichen Grundbegriffes vom Frilbchristentum bis zur Reformation, Münster, 1986.

Feil 2000 : E. Feil (éd.), Streitfall "Religion". Diskussionen zur Bestimmung und Abgrenzung des Religionsbegriffs, Münster, 2000.

Geertz - McCutcheon 2000a : A.W. Geertz, R.T. McCutcheon (éds), Perspectives on Metbod and Theory in the Study of Religion. Adjunct Proceedings of the XVItb Congress of the IAHR, Mexico City, 1995, Leiden, 2000.

Geertz - McCutcheon 2000b : A.W. Geertz, R.T. McCutcheon, "The Role of Method and Theory in the IAHR", in Geertz - McCutcheon 2000a, p. 3-37.

Gladigow 1995: B. Gladigow, "Struktur der Öffentlichkeit und Bekenntnis in polytheistischen Religionen", in H.G. Kippenberg, G.G. Stroumsa (éds), Secrecy' and Concealment. Studies in the History of Meditemanean and Near Eastem Religions, Leiden, 1995, p. 17-35.

Gladigow 1997 : B. Gladigow, "Polytheismus. Akzente, Perpektiven und Optionen der Forschung", Zeitschrift für Religionsgeschichte 5 (1997), p. 69-77.

Headland - Pike - Harris 1990 : Th. N. Headland, K.L. Pike, M. Harris (éds.), Emics and Etics: The Inside/Outside Debate, Newbury Park, 1990.

Idinopoulos - Wilson 1998 : Th.A. Idinopoulos, B.C. Wilson (éds.), What is Religion? Origins, Definitions, and Explanations, Leiden, 1998.

Klimkeit 1997 : H.J. Klimkeit (éd.), Vergleicben und Versteben in der Religionswissenscbaft, Wiesbaden, 1997.

McCutcheon 1995: R.T. McCutcheon, "The Category 'Religion' in Recent Publications: A Critical Survey", Numen 42 (1995), p. 284-309.

McCutcheon 1999 : R.T. McCutcheon (éd.), The Insider Outsider Problem in the Study of Religion. A Reader, London, 1999.

Mora 1994 : F. Mora, "Per una tipologia del politeismo", in Bianchi 1994, p. 823-830.

Mora 2000 : F. Mora, "Verso una tipologia delle religioni classiche", Kernos 13 (2000), p. 9-33.

Niehr 1998: H. Niehr, Religionen in Israels Umwelt. Einfübrung in die nordwestsemitiscben Religionen Syrien-Palästinas, Würzburg, 1998.

Platvoet 1990 : J. Platvoet, "The Definers Defined: Tradition in the Definition of Religion", Method $\varepsilon$ Theory in the Study of Religion 2 (1990), p. 180-211.

Platvoet 1994: J. Platvoet, "Defining the Definers: Non-Verifyability/Non-Falsifiability as a Definiens in an Operational Definition of Religion", in Bianchi 1994, p. 701-712.

Platvoet - Molendijk 1999 : J.G. Platvoet, A.L. Molendijk (éds), The Pragmatics of Defining Religion. Contexts, Concepts and Contests, Leiden, 1999.

Ringgren 1981 : H. Ringgren, Israelitiscbe Religion, Stuttgart, 1981², trad. it. Milano, 1987.

Rudolph 1985a : K. Rudolph, "The Foundations of the History of Religions and Its Future Task", in J.M. Kitagawa (éd.), The History of Religions. Retrospect and Prospect, Chicago, 1985, p. 104120.

Rudolph 1985b : K. Rudolph, Historical Fundamentals and the Study of Religions, New York, 1985. Rudolph 1992 : K. Rudolph, Gescbichte und Probleme der Religionswissenschaft, Leiden, 1992. Sabbatucci 1987 : D. Sabbatucci, Sommario di storia delle religioni, Roma, 1987. 
Sabbatucci 1988 : D. Sabbatucci, "Kultur und Religion", in H. Cancik, B. Gladigow, M. Laubscher (éds), Handbucb religionswissenschaftlicher Gnundbegriffe 1, Stuttgart, 1988, p. 43-58.

Sabbatucci 1990 : D. Sabbatucci, La prospettiva storicorieligiosa, Milano, 1990.

Sabbatucci 1998 : D. Sabbatucci, Politeismo I-II, Roma, 1998.

Saler 1993 : B. Saler, Conceptualizing Religion, Leiden, 1993.

Schmidt 1987 : F. Schmidt (éd.), The Unconceivable Polytbeism. Studies in Religious Historiography, London $1987^{3}$.

Segal - Ryba 1997 : R. Segal, Th. Ryba, "Religion and Post-modernism - a review Symposium", Religion 27 (1997), p. 101-149.

Taylor 1998 : M.C. Taylor (éd.), Critical Terms for Religious Studies, Chicágo/London, 1998.

Van Baaren 1973: Th.P, van Baaren, "Science of Religion as a Systematic Discipline. Some Introductory Remarks", in Th.P. van Baaren, H.J.W. Drijvers (écls), Religion, Culture and Metbodology. Papers of the Groningen Working-group for the Study of Fundamental Problems and Metbods of Science of Religion, The Hague/Paris, 1973, p. 35-56.

Wiebe 1999: D. Wiebe, The Politics of Religious Studies: The Continuing Conflict with Theology in the Academy, New York, 1999.

Xella 1994 : P. Xella, "Le religioni della Siria e della Palestina", in G. Filoramo (éd.), Storia delle religioni I, Bari 1994, p. 219-262. 